\title{
Untargeted Metabolomics Profiling and Global Semi-quantitation of a Prescription Chinese Herbal Medicine Formula Yinqiaosan Using UPLC-QTOF-MS with a Single Exogenous Reference Internal Standard
}

\section{(ㄷ) (ㄱ) (우 $\ominus$}

Authors

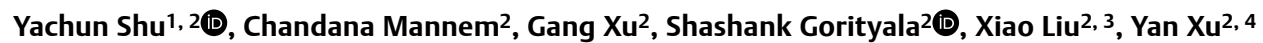

Affiliations

1 Affiliated Hospital of Nanjing University of Chinese Medicine, Nanjing, P. R. China

2 Department of Chemistry, Cleveland State University, Cleveland, $\mathrm{OH}, \mathrm{USA}$

3 College of Pharmacy, Nanjing University of Chinese Medicine, Nanjing, P. R. China

4 Case Comprehensive Cancer Center, Case Western Reserve University, Cleveland, OH, USA

Key words

Yinqiaosan, Chinese herbal medicine formula, untargeted metabolomics profiling, global semiquantitative analysis, multivariate data analysis, UPLC-QTOF-MS

$\begin{array}{ll}\text { received } & 03.09 .2019 \\ \text { revised } & 26.02 .2020 \\ \text { accepted } & 10.03 .2020\end{array}$

\section{Bibliography}

DOI https://doi.org/10.1055/a-1141-0119

Published online: 2020

Planta Med Int Open 2020; 7: e45-e57

(C) Georg Thieme Verlag KG Stuttgart · New York

ISSN 2509-9264

\section{Correspondence}

Yachun Shu

Affiliated Hospital of Nanjing University of Chinese Medicine 155 Hanzhong Road

210029 Nanjing City

jiangsu Province

P. R. China

Tel.: + 8625-86617141, Fax: + 8625-86618555

yfy0093@njucm.edu.cn
Yan Xu

Department of Chemistry, Cleveland State University

2121 Euclid Ave., SI 333

Cleveland

Ohio

44115-2214

USA

Tel.: +1216 687 3991, Fax: + 12166879298

y.xu@csuohio.edu

$\bigoplus$

Supporting Information for this article is available online at: http://www.thieme-connect.de/products.

\begin{abstract}
Yinqiaosan is a classic Chinese herbal medicine formula that has been used to treat various bacterial and viral infections by Chinese medicine doctors for over two centuries. In this work, we developed a comprehensive qualitative and quantitative method for identification, quantitation, and quality assessment of chemical constituents of Yinqiaosan formula in four different preparation forms (i.e., decoction, granule, pill, and tablet), which employed ultra-performance liquid chromatography quadrupole time-of-flight mass spectrometry with a single exogenous reference internal standard for untargeted metabolomics profiling and global semiquantitative analysis. The use of a single exogenous reference internal standard permitted not only qualitative and quantitative analyses of multiple herbal components in a single instrument run, but also crosscomparison of chemical contents in between all four Yinqiaosan preparation forms. The acquired mass chromatograms were analyzed, quantitated, and compared using multivariate data analysis for similarities and differences of chemical constituents in four Yinqiaosan preparation forms. For the first time, we were able to identify over 100 chemical constituents from each preparation form using the available database. Among the 49 commonly identified compounds in the 4 Yinqiaosan preparation forms, 16 have been reported to have pharmacological activities, which may be used in a network pharmacology study of Yinqiaosan for exploring the underlying mechanism of the herbal formula.
\end{abstract}




\section{ABBREVIATIONS}

$\begin{array}{ll}\text { CHM } & \text { Chinese herbal medicine } \\ \text { CV } & \text { coefficient of variation } \\ \text { EBM } & \text { evidence-based medicine } \\ \text { ESI } & \text { electrospray ionization } \\ \text { IS } & \text { internal standard } \\ \text { PCA } & \text { principal component analysis } \\ \text { PLS-DA } & \text { partial least squares discriminant analysis } \\ \text { QC } & \text { quality control } \\ \text { Q-marker } & \text { quality marker } \\ \text { RCT } & \text { randomized controlled trial } \\ \text { SIM } & \text { selected ion monitoring } \\ \text { TCM } & \text { traditional Chinese medicine } \\ \text { TIC } & \text { total ion current } \\ \text { UPLC-QTOF-MS } & \text { ultra-performance liquid chromatogra- } \\ & \text { phy-quadrupole time-of-flight mass } \\ & \text { spectrometry }\end{array}$

\section{Introduction}

CHM formulas are the key components of TCM interventions, which have many preparation forms including decoctions, granules, pills, tablets, capsules, powders, medicated teas, medicated wines, etc. [1]. Despite the widespread use of CHM formulas in Asia and their growing use in the West, the fundamental issue hindering $\mathrm{CHM}$ integration into the Western mainstream health care system is that rigorous scientific evidence of CHM efficacy and safety obtained through RCTs or systematic reviews of RCTs has been limited from EBM perspectives [2]. This is mainly attributed to two major defects in the RCTs to date: (1) improper differentiation or reporting of a TCM pattern or syndrome since pattern differentiation is crucial in TCM for prescribing CHM therapy, and (2) the lack of a comprehensive quality assessment and control of CHM formulas since the chemical constituents in medicinal plants vary with geographic origin and cultivar, parts in use, time of harvest, ecological environment and potential contamination with heavy metals, pesticides, or mycotoxins, processing and detoxification method as well as various forms of preparations [1].

The methods currently adopted by the international pharmacopeial monographs [3-5] for identification and quality assessment of medicinal plants and CHM formulas emphasize the qualitative and quantitative determination of a group of subjectively selected Q-marker constituents that may or may not have a therapeutic effect. These methods can't distinguish whether a marker constituent is endogenous or falsified because no information on qualitative and quantitative chemical profiles of medicinal plants or $\mathrm{CHM}$ formulas is obtained. Therefore, comprehensive qualitative and quantitative chemical analysis methods are much more desirable, not only for phytochemical profiling and quality assessment of medicinal plants and CHM formulas but also for detection of counterfeits. Untargeted metabolomics profiling using LC-QTOF-MS has been successfully applied to fingerprinting herbal medicines [6-8]. If this technique combines with the use of a single exogenous reference IS for semiquantitative analysis and PCA for pattern recog- nition, it would provide a more adequate method for identification of chemical constituents and quality assessment of medicinal plants and CHM formulas. This work demonstrates such an attempt.

Yinqiaosan is a classic CHM formula developed by the Qing Dynasty famous Chinese doctor Wu Jutong (1758-1836). It is composed of nine herbs including Jinyinhua (Flos lonicerae), Lianqiao (Fructus forsythiae), Bohe (Herba menthae), Jingjie (Herba schizonepetae), Niubangzhi (Fructus arctii), Jiegeng (Radix platycodonis), Dandouchi (Semen Sojae preparatum), Gancao (Radix glycyrrhizae), and Danzhuye (Herba lophatheri) [9]. Based on CHM classification, Yinqiaosan has cool energy, an acrid taste, and properties of releasing exterior, clearing heat and removing toxicity from human bodies [10]. It has been used by TCM doctors for over two centuries as treatment for various bacterial and viral infections such as influenza [11], hand, foot, and mouth disease [12], pharyngitis [13], pneumonia [14], acute tonsillitis [15], measles [16], and mumps [17]. A recent randomized clinical trial indicated that Yinqiaosan plus another CHM formula, Maxingshigan, was as effective as oseltamivir (also known as Tamiflu) for the treatment of H1N1 influenza A virus infections [18].

Yinqiaosan was originally prepared in the form of a decoction. Due to the ease of use, carry, and storage, Yinqiaosan has been prepared in various forms over the course of history, such as Yinqiaosan peifangkeli (granule), Yinqiaojieduwan (pill), and Yinqiaojiedupian (tablet), which are being used interchangeably in clinical interventions. Currently, there are various forms of Yinqiaosan produced by numerous Chinese manufacturers [19]. The lack of comprehensive qualitative and quantitative chemical analysis methods for chemical profiling and quantitation not only impede complete quality assessment of various Yinqiaosan preparation forms, but also confuse the assessment of outcomes of clinical studies [2,20]. Therefore, we have developed a comprehensive qualitative and quantitative method that combines untargeted metabolomics profiling and global semiquantitative analysis with the use of a single exogenous IS, as well as pattern recognition through PCA for identification, quantitation, and quality assessment of Yinqiaosan in four preparation forms (decoction, granule, pill, and tablet).

\section{Results and Discussion}

One of the key technical advances of the method developed is the implementation of a single exogenous reference IS (etoposide-d3), which serves multiple roles including selection of retention time and mass shift windows for UPLC-QTOF-MS analysis, assessement and correction of a sample matrix effect, peak normalization for multivariate data analysis, and global semiquantitative analysis of multicomponents in each Yinqiaosan preparation form, as well as cross-comparison of chemical contents in between various Yinqiaosan preparation forms.

Our experimental data (not shown) indicated that there were no chromatographic or mass spectrometric interferences to the IS in the blank solutions and samples of the four Yinqiaosan preparation forms. The matrix effect of each Yinqiaosan preparation form on the mass spectrometric detection of the IS expressed as matrix factor was calculated by the mean peak area of the IS spiked in samples of each Yinqiaosan preparation form over that of the IS spiked 
- Table 1 Matrix effects of Yinqiaosan samples on mass spectrometric detection of the ISa.

\begin{tabular}{|l|l|l|l|l|}
\hline Sample matrix $(\mathbf{n}=\mathbf{3})$ & $\mathbf{E S I}$ mode & $\mathbf{P A}_{\mathbf{I S}}{ }^{\mathbf{b}}$ in extracted sample matrix $\pm \mathbf{S D} \mathbf{c}$ & $\mathbf{P A}_{\mathbf{I S}}$ in solution $\pm \mathbf{S D}$ & $\mathbf{M F}_{\mathbf{I S}} \mathbf{d} \pm \mathbf{S D}$ \\
\hline \multirow{3}{*}{ Decoction } & + & $(4.75 \pm 0.06) \times 10^{5}$ & $(5.4 \pm 0.2) \times 10^{5}$ & $0.88 \pm 0.03$ \\
\cline { 2 - 6 } & - & $(2.34 \pm 0.02) \times 10^{6}$ & $(2.68 \pm 0.03) \times 10^{6}$ & $0.87 \pm 0.01$ \\
\hline \multirow{2}{*}{ Granule } & + & $(5.4 \pm 0.2) \times 10^{5}$ & $(5.4 \pm 0.2) \times 10^{5}$ & $1.00 \pm 0.05$ \\
\cline { 2 - 6 } & - & $(2.52 \pm 0.03) \times 10^{6}$ & $(2.68 \pm 0.03) \times 10^{6}$ & $0.94 \pm 0.02$ \\
\hline \multirow{2}{*}{ Tablet } & + & $(5.8 \pm 0.2) \times 10^{5}$ & $(2.6 \pm 0.2) \times 10^{5}$ & $1.07 \pm 0.05$ \\
\cline { 2 - 6 } & - & $(2.37 \pm 0.04) \times 10^{6}$ & $(5.4 \pm 0.2) \times 10^{5}$ & $0.88 \pm 0.02$ \\
\hline \multirow{2}{*}{ Pill } & + & $(4.83 \pm 0.04) \times 10^{5}$ & $(2.68 \pm 0.03) \times 10^{6}$ & $0.89 \pm 0.03$ \\
\cline { 2 - 6 } & - & $(2.43 \pm 0.01) \times 10^{6}$ & & $0.91 \pm 0.01$ \\
\hline
\end{tabular}

$\mathrm{a} I S=1.69 \mu \mathrm{M},{ }^{\mathrm{b}} \mathrm{A}_{\mathrm{IS}}=$ mean peak area of the spiked IS, ${ }^{\mathrm{CSD}}=$ standard deviation, ${ }^{\mathrm{d}} \mathrm{MF}_{\mathrm{IS}}=\left(\mathrm{PA}_{\mathrm{IS}}\right.$ in the extracted sample matrix $) /\left(\mathrm{PA}_{\mathrm{IS}}\right.$ in the solution $)$.
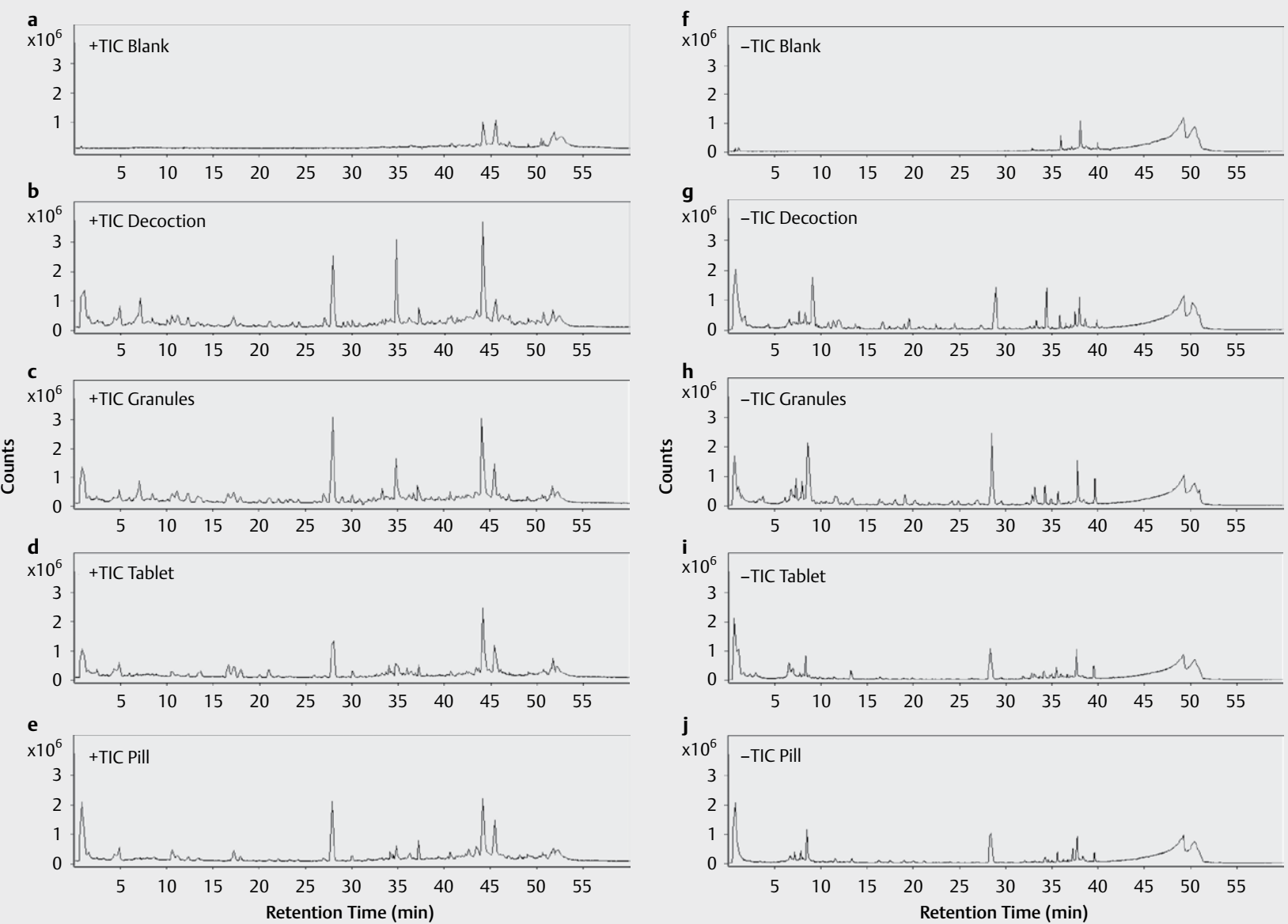

- Fig. 1 Representative TIC chromatograms of the solution blank and samples of various Yinqiaosan preparation forms by both positive ionization mode (a-e) and negative ionization mode $(\mathbf{f}-\mathbf{j})$.

in the blank solutions. The matrix factors of four Yinqiaosan preparation forms ranged from 0.87-1.07 ( Table 1), indicating there was no significant signal suppression or enhancement of the IS in any of the sample matrices.

For untargeted metabolomics profiling, constituent identification, and global semiquantitation of chemical constituents in Yinqiaosan formula, triplicate samples were prepared for each Yinqiaosan preparation form and the solution blank. A total of 15 Yinqiaosan samples were analyzed using the UPLC-QTOF-MS method developed. The chromatographic and spectra (MS and MS/MS) data were acquired by both positive and negative ESI modes. The representative TIC chromatograms are shown in \ Fig. $\mathbf{1}$. Chemical constituents of each Yinqiaosan preparation form were extracted after subtracting TIC chromatograms of the solution blanks from those of Yinqiaosan sample solutions.

There are several nonvolatile, water-soluble Q-markers from the nine herbs of Yinqiaosan [3]. To illustrate this targeted feature of the method developed, these Q-markers were targeted and ex- 


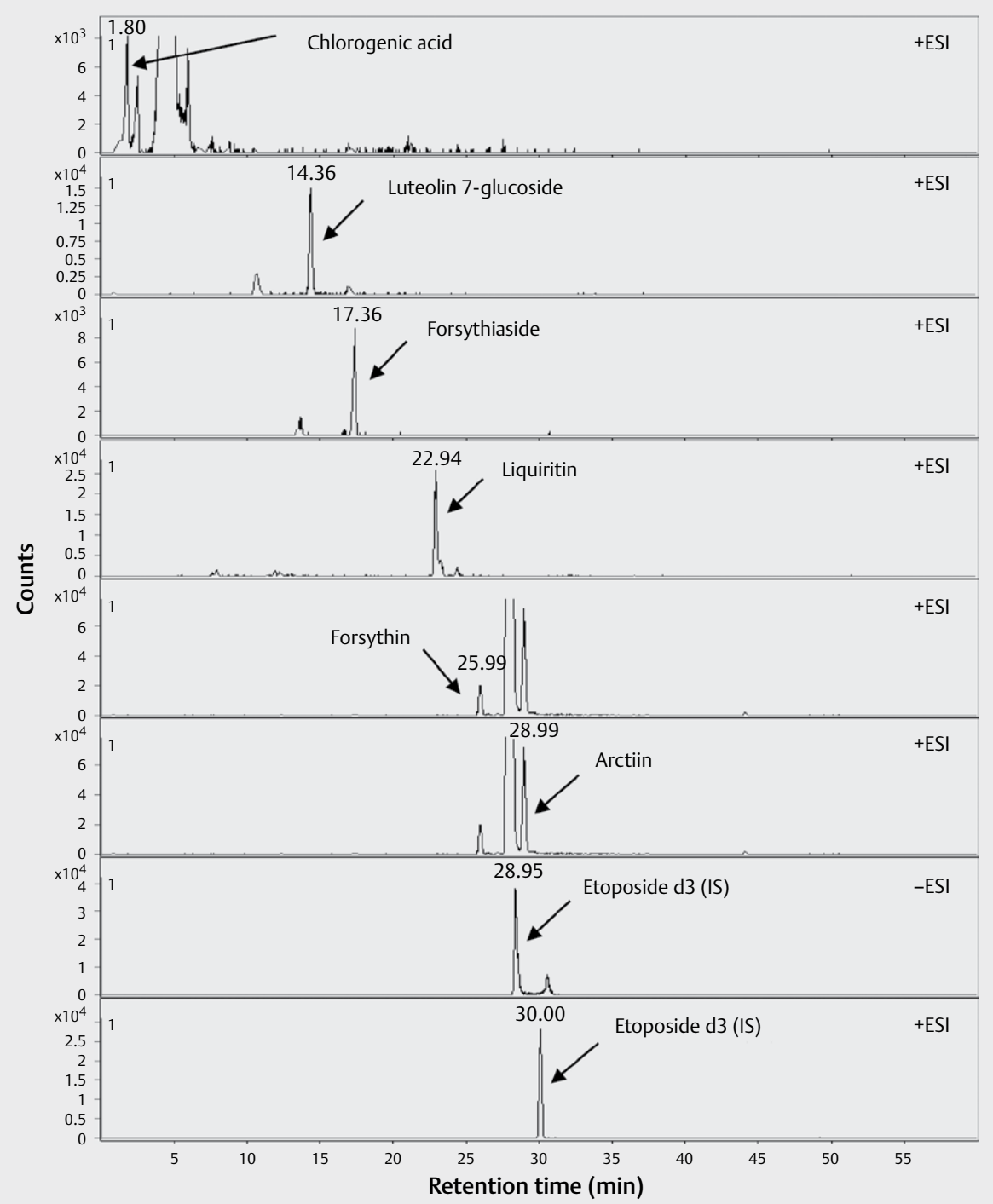

Fig. 2 Representative SIM chromatograms of six marker constituents of Yinqiaosan along with the IS at a concentration of $1.69 \mu \mathrm{M}$.

tracted. The representative SIM chromatograms of six Q-markers (i.e., chlorogenic acid, luteolin 7-glucoside, forsythiaside, liquiritin, forsythin, and arctiin) are provided in > Fig. 2.

Due to the shortage of standard reference material and the cost concern, determination of multicomponents for quality assessment of CHM formula is currently not required by the Chinese regulatory agency. In the case of Yinqiaosan products, only one of the Q-markers (i.e., arctiin, chlorogenic acid, or forsythin) is regulated by the Chinese Pharmacopoeia [3]. Since Yinqiaosan products contain multiple bioactive components, the current methods for single-component analysis can neither assess the quality of Yinqiaosan products objectively nor detect counterfeits effectively. The method developed in this work provides a much better technical solution for quality assessment of Yinqiaosan products, which not only profiles a product but also quantitates multiple Q-markers simultaneously.

Identification of chemical constituents in each Yinqiaosan preparation form was accomplished by following the procedure detailed in "Data processing, constituent identification, and statistical analysis" in the "Materials and Methods" section. The data obtained were then subjected to molecular feature extractions using possible ion adducts, isotope patterns, and charge states as well as a preset retention time and mass window. It is worth noting that the post-processing filter of the data processing software was set at 3 out of 3 to ensure each molecular feature extracted presented in all three mass chromatograms of the triplicate measurements for each Yinqiaosan preparation form. The total number of constituents identified with chemical names and the number of constituents identified with molecular formulas in each preparation form are summarized in $>$ Table 2 . The number of chemical constituents found in decoction, granule, pill, and tablet forms of Yinqiaosan were $302,434,427$, and 388, respectively. Among the 4 preparation forms, there were 104 common constituents (> Fig. 3), 49 identified with both chemical names and formulas ( $>$ Table 3 ) and 55 identified only with chemical formulas ( $\triangleright$ Table 1S, Supporting Information). The MS/MS spectra of the 49 chemical constituents 
- Table 2 Summary of chemical constituents found in four Yinqiaosan preparation forms.

\begin{tabular}{|c|c|c|c|c|c|c|c|c|}
\hline \multirow{2}{*}{$\begin{array}{l}\text { Yinqiaosan } \\
\text { Forms }\end{array}$} & \multicolumn{4}{|c|}{ Constituents } & \multicolumn{4}{|c|}{ Common constituents } \\
\hline & ESI mode & Identified & Unidentified & Total & ESI mode & Identified & Unidentified & Total \\
\hline \multirow{2}{*}{ Decoction } & + & 158 & 37 & \multirow{2}{*}{302} & \multirow{4}{*}{+} & \multirow{4}{*}{46} & \multirow{4}{*}{21} & \multirow{8}{*}{104} \\
\hline & - & 21 & 86 & & & & & \\
\hline \multirow{2}{*}{ Granule } & + & 79 & 177 & \multirow{2}{*}{434} & & & & \\
\hline & - & 36 & 142 & & & & & \\
\hline \multirow{2}{*}{ Tablet } & + & 91 & 163 & \multirow{2}{*}{388} & \multirow{4}{*}{-} & \multirow{4}{*}{3} & \multirow{4}{*}{34} & \\
\hline & - & 24 & 110 & & & & & \\
\hline \multirow[t]{2}{*}{ Pill } & + & 67 & 186 & \multirow{2}{*}{427} & & & & \\
\hline & - & 38 & 136 & & & & & \\
\hline
\end{tabular}

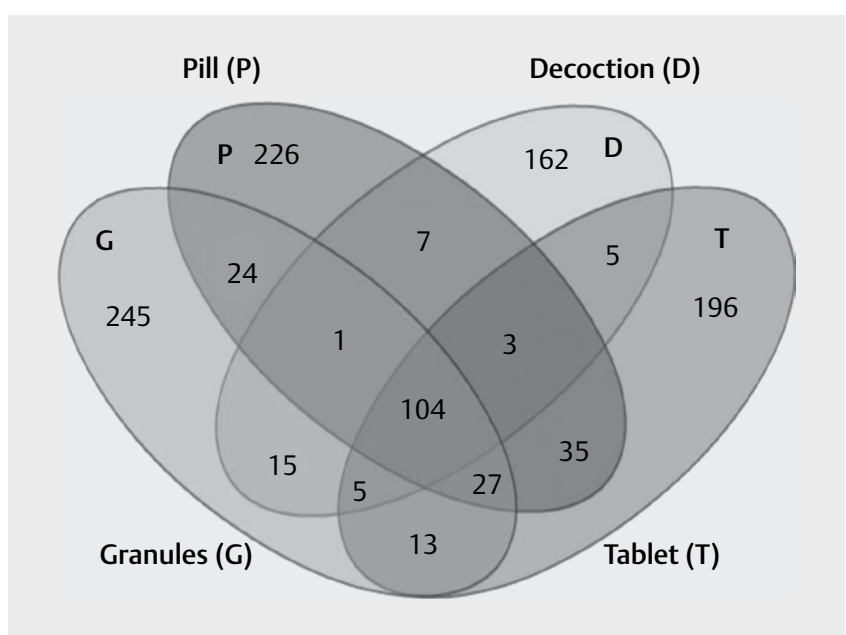

- Fig. 3 Venn diagram of the chemical constituents found in each preparation form of Yinqiaosan.

commonly identified in all four Yinqiaosan preparation forms are provided in ( $\triangleright$ Fig. 1S, Supporting Information).

Since each Yinqiaosan preparation form exerts a similar therapeutic effect and is used to treat the same illnesses, it is reasonable to think that pharmacologically active constituents are among the 49 commonly identified chemical constituents in all four preparation forms, whereas the unique chemical constituents in each preparation form ( $\triangleright$ Tables 2S-9S, Supporting Information) may come from the herbs used by the manufacturers, where chemical constituents may vary with geographic origin and cultivar, time of harvest and the ecological environment of the herbs, potential agricultural pollutions of pesticides and herbicides or industrial crosscontamination with other pharmaceuticals as well as side reactions and by-products associated with the unique manufacturing conditions (e.g., temperature, pressure, time, solvent, additives, etc.) of each Yinqiaosan preparation form. This study shows that product profiling is an indispensable part of quality assessment, and the untargeted metabolomics approach not only allows us to profile but also fingerprint an herbal product in terms of Q-markers, origin and cultivars, potential contaminations, and signature constituents associated with a particular preparation or manufacturing process.
Multivariate analysis of the acquired MS data (i.e., exact mass to retention time pair and normalized peak area) was first carried out with an unsupervised PCA score plot to assess the similarities of chemical constituents among the four Yinqiaosan preparation forms and the reproducibility of replicate samples of each preparation form by the UPLC-QTOF-MS method, then visualized by a supervised PLS-DA score plot to establish recognition patterns of the four Yinqiaosan preparation forms.

As shown in the PCA score plot ( $\triangleright$ Fig. 4a), the differences in chemical constituents among the four Yinqiaosan preparation forms were apparent. The first two principal components encompassed $72.3 \%$ of the total variance, and the tight grouping of triplicate measurements on the samples of each preparation form indicated that the UPLC-QTOF-MS method developed had excellent reproducibility. The PLS-DA score plot ( $\mathbf{F i g . 4 b}$ ) was in agreement with the PCA score plot, and showed distinctive patterns among the four Yinqiaosan preparation forms. Hence, these patterns may be used for product differentiation and recognition.

A high-throughput UPLC-QTOF-MS method for global semiquantitative analysis of chemical constituents in CHM formula was demonstrated in this work. As illustrated by the contents of 49 commonly identified chemical constituents in four Yinqiaosan preparation forms ( $\triangleright$ Table 4 ), the use of a single exogenous reference IS (etoposide-d3) in the method enabled us to perform not only global semiquantitative analysis of multicomponents in each Yinqiaosan preparation form but also cross-comparison among the four preparation forms. Therefore, the method developed provides an efficient and economical solution for both herbal content analysis and product comparison.

The reproducibility of global semiquantitative analysis by the UPLC-QTOF-MS method was investigated by triplicate measurements of sample of each Yinqiaosan preparation form, and the precision of the semiquantitative measurements was calculated in terms of CV of the chemical contents. It was shown ( $\vee$ Table 4 ) that 91.8-98.0\% of the 49 chemical constituents of the 4 preparation forms had CV values that fell within the recommended values ( $C V \leq$ $30 \%$ ) [21], which indicated the reliability of the UPLC-QTOF-MS method.

The pharmacological activities of the 49 commonly identified chemical constituents in all 4 Yinqiaosan preparation forms were investigated through text mining and database searching. Sixteen of the forty-nine chemical constituents were found to have various 

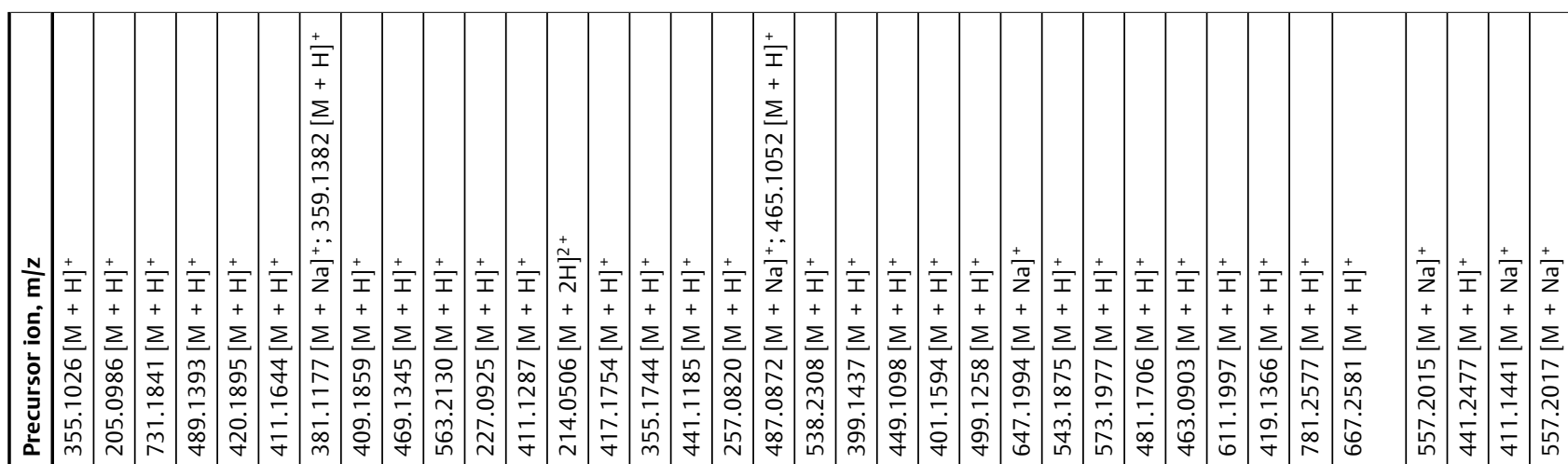

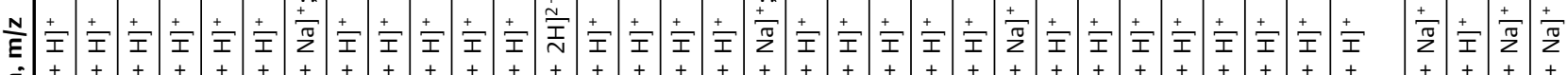

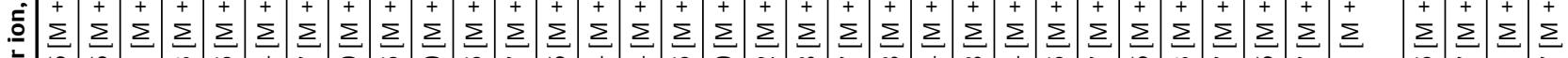

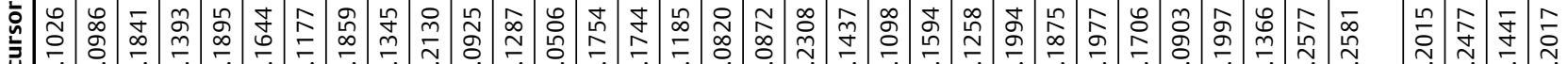

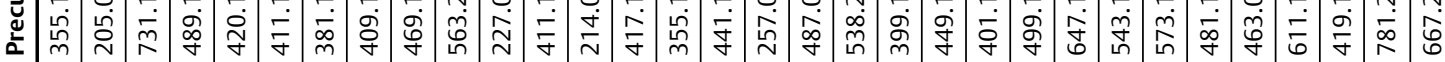

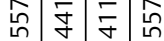

蒫

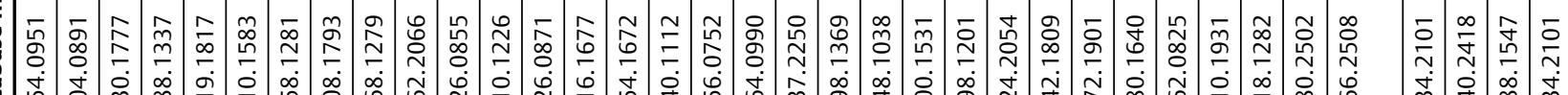
Dٓ̃

气ี

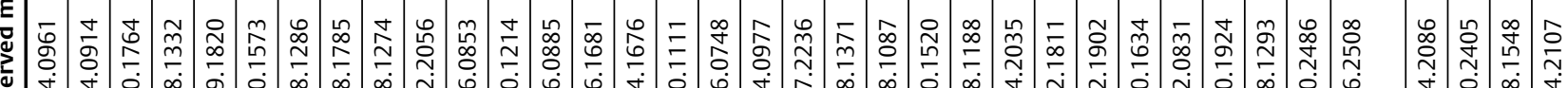

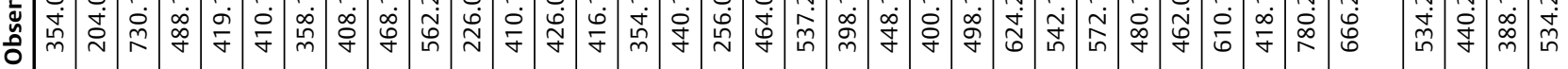

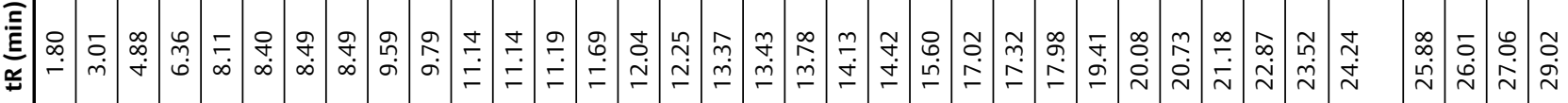

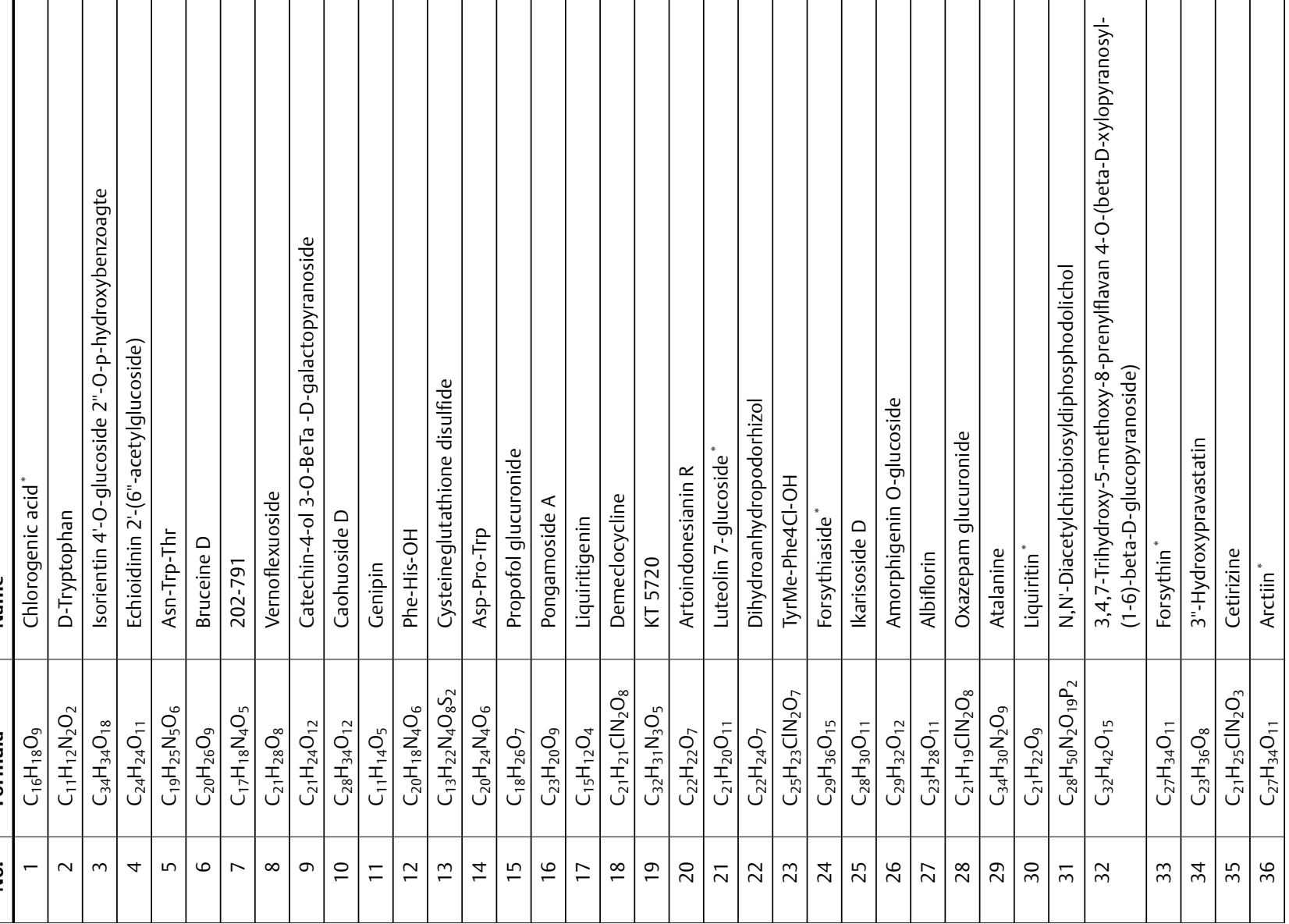




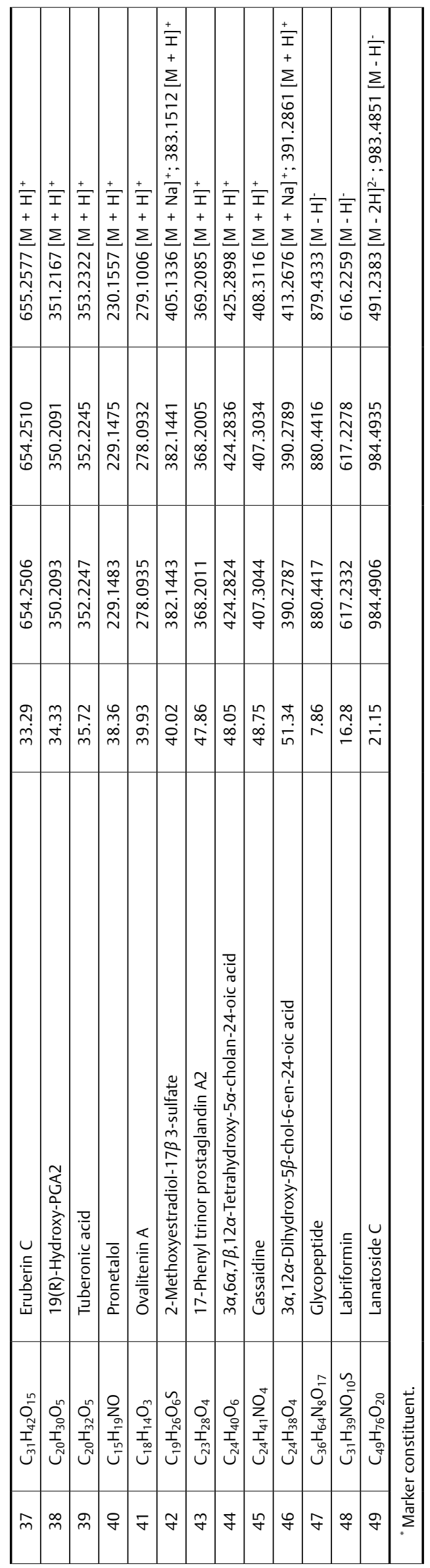

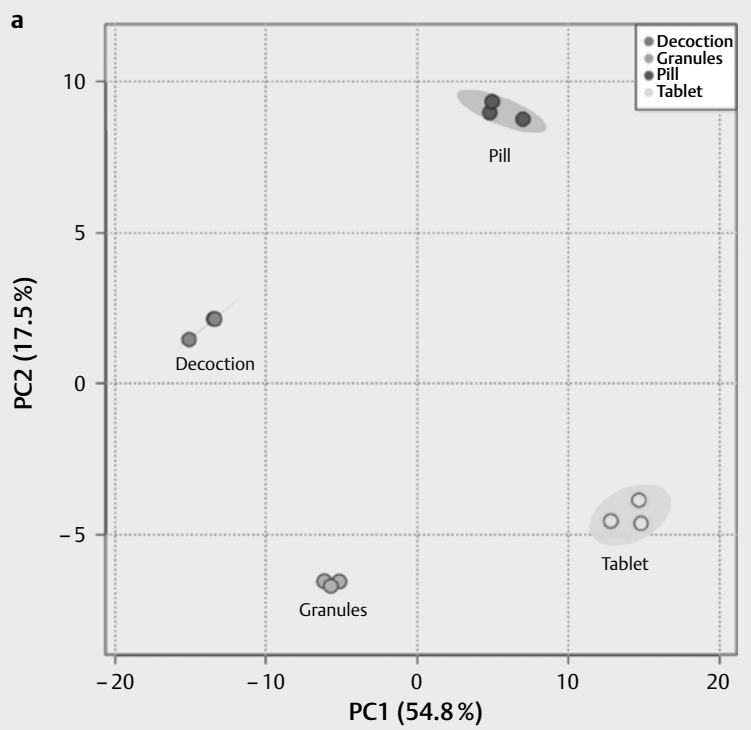

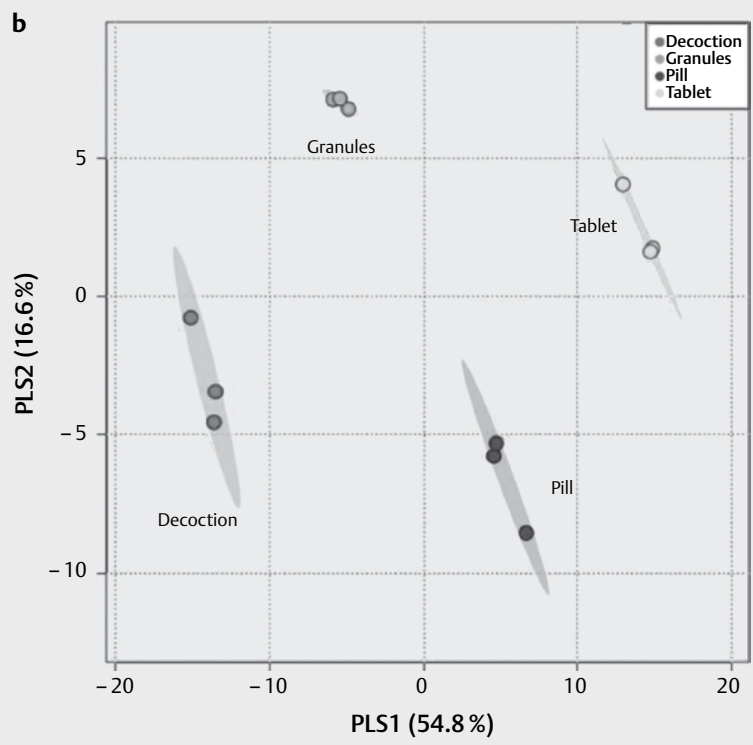

- Fig. 4 Visualization of multivariate data analysis. a The PCA score plot, and $\mathbf{b}$ the PLS-DA score plot of four Yinqiaosan preparation forms.

pharmacological activities ( $\$$ Table 5), ranging from antibacterial, antiviral, anti-inflammatory, and antifungal to antioxidant, anticancer, and treatment of allergic rhinitis [22-52]. These pharmacological active compounds may serve not only as lead compounds in new drug development, but also as bait for the retrieval of protein targets in a network pharmacology study $[53,54]$. The latter may help us to understand the underlying action mechanism of Yinqiaosan formula.

In conclusion, a UPLC-QTOF-MS method with the use of a single exogenous reference IS has been developed for untargeted metabolomics profiling and global semiquantitation of the prescription Chinese herbal medicine formula Yinqiaosan. The chemical profiles of 4 Yinqiaosan preparation forms (i.e., decoction, granule, pill, and 


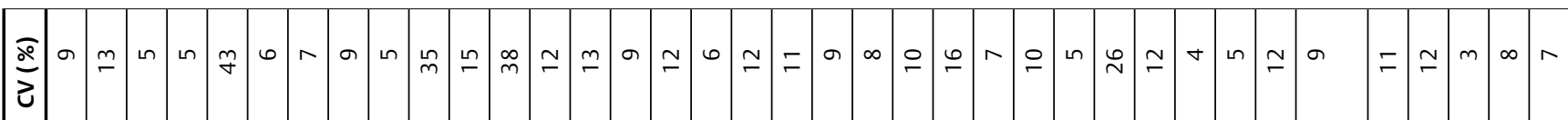

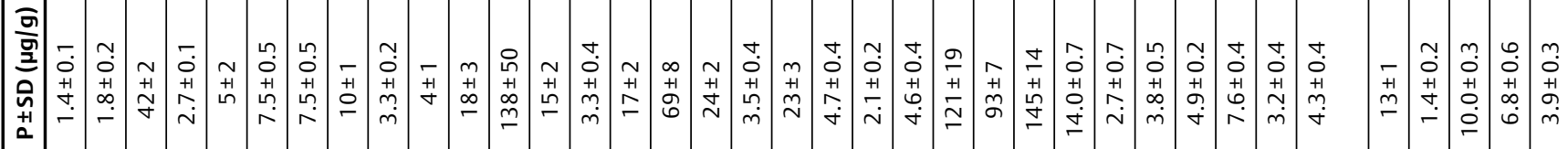

$\int \frac{\partial}{\rho}$

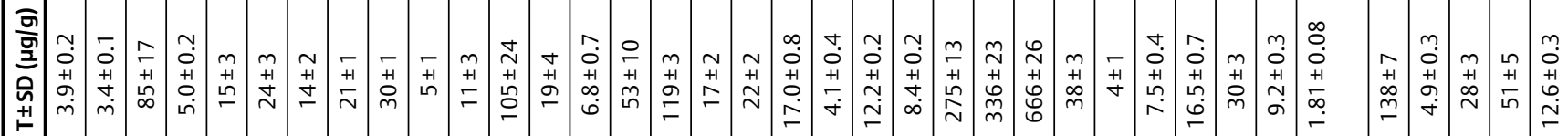

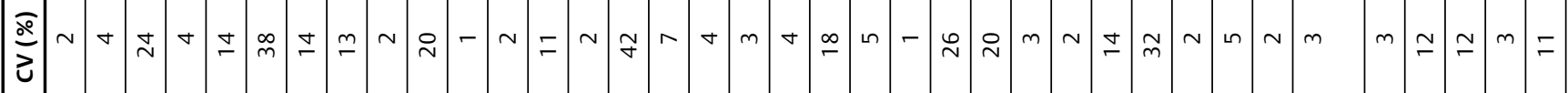

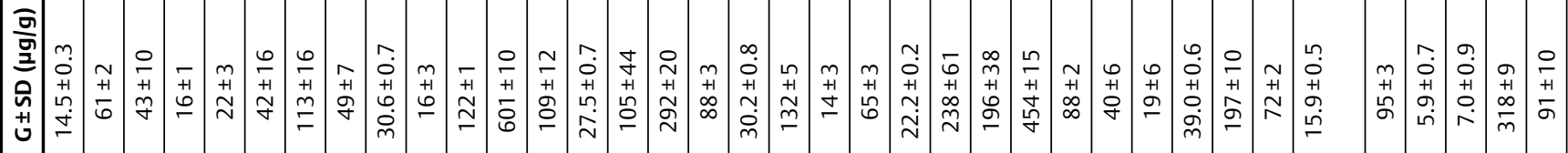

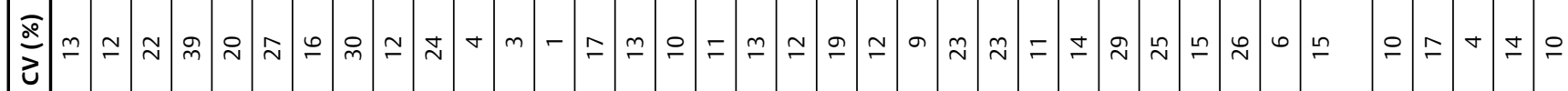

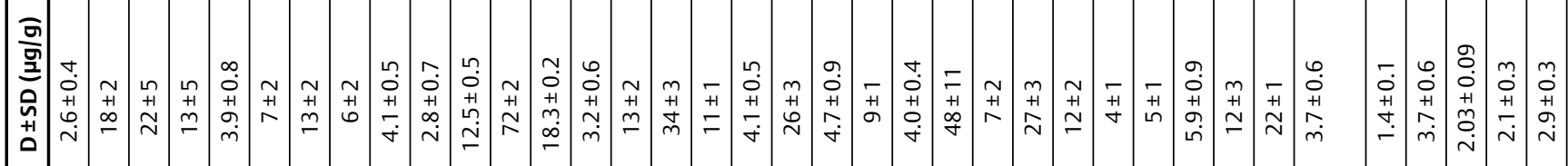

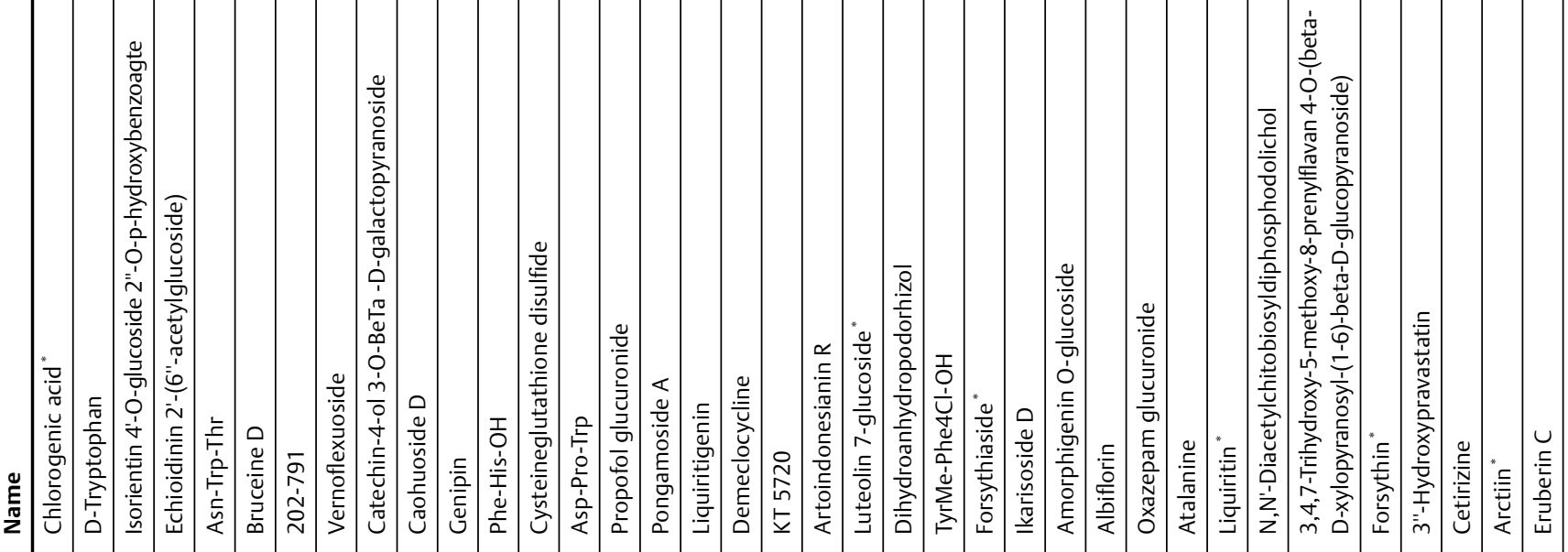

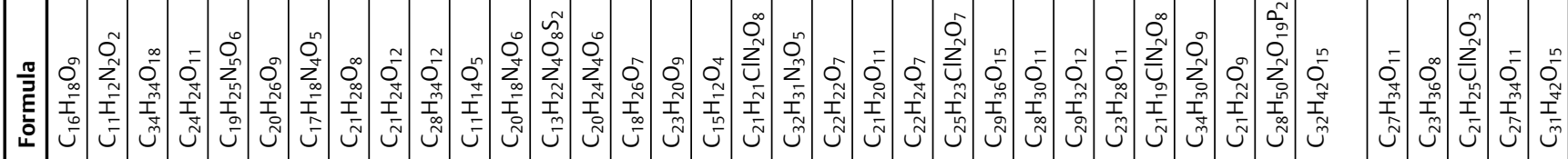

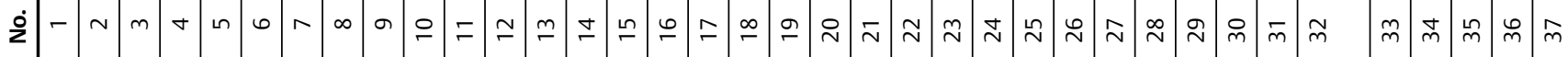




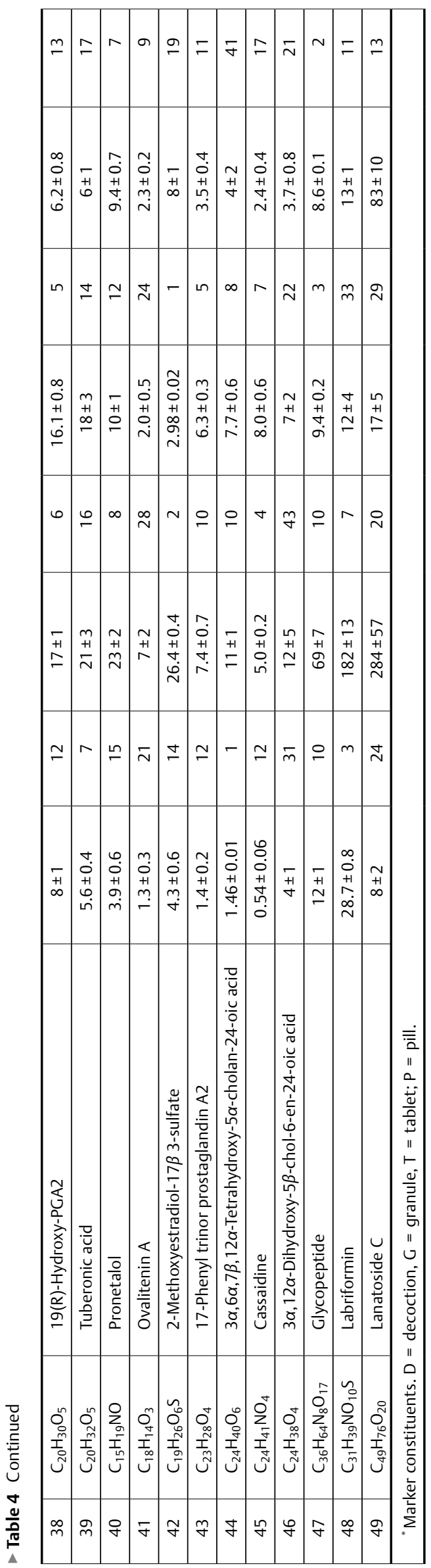

tablet) were obtained, and the 49 common chemical constituents and 16 pharmacological active compounds were identified. Simultaneous semiquantitative analysis of the multicomponents in each Yinqiaosan preparation form was carried out along with untargeted metabolomics profiling, and cross-comparison of the chemical contents in between four preparation forms was accomplished. PCA and PLS-DA analyses showed that the UPLC-QTOF-MS method developed was reproducible and the chemical constituents found in the four Yinqiaosan preparation forms displayed unique patterns for product differentiation and recognition. The method developed is useful for the identification, quantitation, and cross-comparison of chemical constituents not only in Yinqiaosan products, but also in other CHM formulas.

\section{Materials and Methods}

\section{Chemicals and Chinese herbal medicine formulas}

Ammonium hydroxide and formic acid were purchased from Sigma-Aldrich. Optima LC/MS grade acetonitrile and methanol, and HPLC grade water were from Fisher Scientific. Deionized water was obtained from an in-house Barnstead Nanopuree water purification system (Thermo Scientific) with a resistivity meter reading of 18.2 M $\Omega$-cm. Etoposide-d3 (purity, 97.8\%) was purchased from Toronto Research Chemicals and used as the single exogenous reference internal standard in this work.

The Yinqiaosan decoction was prepared by Jiangsu Provincial Hospital of Traditional Chinese Medicine (Nanjing, Jiangsu, China) with nine kinds of processed CHMs (Jinyinhua, Batch No. 150320; Lianqiao, Batch No. 150718; Bohe, Batch No. 150701; jingjie, Batch No. 150313; Niubangzhi, Batch No. 150601; Jiegeng, Batch No. 150601; Dandouchi, Batch No. 150401; Gancao, Batch No. 150701; and Danzhuye, Batch No. 150302). Yinqiaosan peifangkeli (granules) were manufactured by jiangyin Tianjiang Pharmaceutical Co. (Jiangyin, Jiangsu, PRC) as individual packages for each CHM [i.e., jinyinhua (Batch No. 1503112), Lianqiao (Batch No. 1501092), Bohe (Batch No. 1412140), Jingjie (Batch No. 1501094), Niubangzhi (Batch No. 1411156), Jiegeng (Batch No. 1503133), Dandouchi (Batch No. 1410023), Gancao (Batch No. 1502100), and Danzhuye (Batch No. 1501021)]. Yinqiaojieduwan (pills) (Batch No. 14033352) was produced by Beijing Tongrentang (Fengtai District, Beijing, PRC). Yinqiaojiedupian (tablets) (Batch No. 140695) was made by the Yunnan Tengyao Pharmaceutical Co. (Tengyue, Yunnan, PRC).

\section{Preparation of the internal standard solution}

The stock solution of IS was prepared by dissolving $1.00 \mathrm{mg}$ etoposide-d3 powder in $1.00 \mathrm{~mL}$ of methanol to a concentration of 1.00 $\mathrm{mg} / \mathrm{mL}$. The working solution of the IS was prepared by a $1 / 10 \mathrm{di}-$ lution of the stock solution in methanol to a concentration of 100 $\mu \mathrm{g} / \mathrm{mL}$ (or $169 \mu \mathrm{M}$ ).

\section{Preparation of various forms of Yinqiaosan sample solutions}

Yinqiaosan decoction sample solution

The Yinqiaosan decoction was prepared using the following procedure: First, seven of the nine processed CHMs, Jinyinhua (Flos Lonicerae, $9.00 \mathrm{~g}$ ), Lianqiao (Fructus Forsythiae, $9.00 \mathrm{~g}$ ), Niubangzhi 
- Table 5 Pharmacological active constituents found in all four Yinqiaosan preparation forms.

\begin{tabular}{|c|l|c|l|l|}
\hline No. & Name & PubChem CID & CAS & Reported pharmacological activity \\
\hline 1 & Chlorogenic acid $^{*}$ & 1794427 & $327-97-9$ & antibacterial, antiviral [26-27] \\
\hline 2 & Luteolin 7-glucoside $^{*}$ & 5280637 & $5373-11-5$ & antibacterial, antifungal, antioxidant, anti-inflammatory [28, 29], antidiabetic [30] \\
\hline 3 & Forsythiaside $^{*}$ & 5281773 & $79916-77-1$ & antibacterial, antiviral, anti-inflammatory [31-32] \\
\hline 4 & Forsythin $^{*}$ & 101712 & $487-41-2$ & antioxidant, anti-inflammatory [33] \\
\hline 5 & Liquiritin $^{*}$ & 503737 & $551-15-5$ & antioxidant [34], antioxidative, anticancer, and neuroprotective [35] \\
\hline 6 & Arctiin $^{*}$ & 100528 & $20362-31-6$ & antiviral, anti-inflammatory [36-39] \\
\hline 7 & D-Tryptophan & 9060 & $153-94-6$ & antibacterial [40] \\
\hline 8 & Bruceine D $^{*}$ & 441788 & $21499-66-1$ & anticancer: inhibits hepatocellular carcinoma growth [41] \\
\hline 9 & 202-791 & 122114 & $101342-80-7$ & a calcium channel agonist [42] \\
\hline 10 & Genipin & 442424 & $6902-77-8$ & anticancer: suppresses colorectal cancer cells [43-45] \\
\hline 11 & Liquiritigenin & 114829 & $578-86-9$ & $\begin{array}{l}\text { restores osteoblast damage [46], attenuates cardiac injury [47], prevents } \\
\text { palmitate-induced beta-cell apoptosis [48] }\end{array}$ \\
\hline 12 & Demeclocycline & 54680690 & $127-33-3$ & for the treatment of hyponatremia secondary to SIADH [49] \\
\hline 13 & KT 5720 & 3844 & $108068-98-0$ & reverses multidrug resistance, a chemosensitizer [50-51] \\
\hline 14 & Albiflorin & 51346141 & $39011-90-0$ & ameliorates obesity [52] \\
\hline 15 & Cetirizine & 2678 & $83881-51-0$ & for the treatment of allergic rhinitis [53-54] \\
\hline 16 & Lanatoside C & 656630 & $17575-22-3$ & $\begin{array}{l}\text { anticancer: induces apoptosis in human gastric cancer cells [55] and human } \\
\text { hepatocellular carcinoma cells [56] }\end{array}$ \\
\hline${ }^{*}$ Marker constituents. & & & \\
\hline & & & & \\
\hline
\end{tabular}

(Fructus Arctii, $9.00 \mathrm{~g}$ ), Jiegeng (Radix Platycodonis, $6.00 \mathrm{~g}$ ), Dandouchi (Semen Sojae Preparatum, $5.00 \mathrm{~g}$ ), Gancao (Radix Glycyrrhizae, $5.00 \mathrm{~g}$ ), and Danzhuye (Herba Lophatheri, $4.00 \mathrm{~g}$ ) were first soaked in $696 \mathrm{~mL}$ of deionized water inside a clay pot for $30 \mathrm{~min}$; next, the pot was heated over a gas range with a high flame. Once the water inside the pot started to boil, the gas flame was adjusted to low and the pot continued to be heated for another $10 \mathrm{~min}$. At this point, the remaining two processed CHMs, Bohe (Herba Menthae, $6.00 \mathrm{~g}$ ) and Jingjie (Herba Schizonepetae, $5.00 \mathrm{~g}$ ), were added, and boiling was continued for another $5 \mathrm{~min}$. At the end of heating, the hot decoction $(<500 \mathrm{~mL})$ was poured into a $500-\mathrm{mL}$ glass beaker and cooled to room temperature. The cooled decoction was transferred into a $500-\mathrm{mL}$ volumetric flask, and the beaker was rinsed three times with deionized water. The rinse solution was then combined with the decoction in the flask, and additional deionized water was added to the $500-\mathrm{mL}$ mark of the flask. The solution was vortexed and allowed to settle on a benchtop for 30 $\mathrm{min}$; then, $3.00 \mathrm{~mL}$ of the supernatant were pipetted and transferred into a borosilicate glass tube $(16 \times 100 \mathrm{~mm}$; Fisher Scientific) followed by the addition of $6.90 \mathrm{~mL}$ of methanol and $0.100 \mathrm{~mL}$ of the IS working solution $(169 \mu \mathrm{M})$. The mixture was vortexed for 30 s using a MaxiMix II Vortex Mixer (Thermo Scientific). Next, 1.00 $\mathrm{mL}$ of the solution was transferred into a $1.5-\mathrm{mL}$ microcentrifuge tube (VWR), and centrifuged at $18000 \times \mathrm{g}$ for $10 \mathrm{~min}$ using a Sorvall ST 40R centrifuge (ThermoFisher Scientific). After centrifugation, $500 \mu \mathrm{L}$ of the supernatant was transferred into an 8-mm clear glass screw thread autosampler vial from ThermoFisher Scientific and subjected to UPLC-QTOF-MS analysis.

Yinqiaosan granule, pill, and tablet sample solutions

First, $1 / 12.5$ of the daily maximum dose of each preparation form of Yinqiaosan was weighed out precisely, and each sample was transferred into a 50.0-mL centrifuge tube (Corning). Then, deionized water was added to the $40.0-\mathrm{mL}$ mark. Each sample was soaked in deionized water for $90 \mathrm{~min}$, followed by vortex mixing for $3 \mathrm{~min}$, then placed in a ThermoFisher Scientific FS-28 ultrasonic bath for $30 \mathrm{~min}$. After settling on a benchtop for $30 \mathrm{~min}, 3.00 \mathrm{~mL}$ of the supernatant were pipetted and transferred into a borosilicate glass tube $(16 \times 100 \mathrm{~mm})$, and then $6.90 \mathrm{~mL}$ of methanol and $0.100 \mathrm{~mL}$ of the IS working solution $(169 \mu \mathrm{M})$ were added. The mixture was vortexed for $30 \mathrm{~s}$. Next, $1.00 \mathrm{~mL}$ of the solution was transferred into a $1.5-\mathrm{mL}$ microcentrifuge tube and centrifuged at $18000 \times \mathrm{g}$ for 10 min. After centrifugation, $500 \mu \mathrm{L}$ of the supernatant were transferred into an 8-mm clear glass screw thread autosampler vial and subjected to UPLC-QTOF-MS analysis.

\section{Preparation of QC sample}

The QC sample $(800 \mu \mathrm{L})$ could be prepared by mixing $200 \mu \mathrm{L}$ of each of the four Yinqiaosan sample solutions (see "Preparation of various forms of Yinqiaosan sample solutions"), and used with each batch analysis by monitoring the selectivity and reproducibility of the method over the course of analysis on the 49 commonly identified compounds.

\section{UPLC-QTOF-MS system}

The UPLC-QTOF-MS system used in this work consisted of an Agilent 1290 Infinity UPLC system coupled with an Agilent 6540 QTOF mass spectrometer. The UPLC system included a solvent reservoir, a degasser, a G4220A binary pump, a G1330B thermostat, a G4226A autosampler, a G1316C thermostatted column compartment, and a G4212A diode array detector. The mass spectrometer was equipped with an Agilent jet stream ESI probe.

Liquid chromatographic separation was achieved using gradient elution on a Waters ACQUITY UPLC BEH C18 (2.1 mm i.d. × $100 \mathrm{~mm}$, 
$1.7 \mu \mathrm{m}, 130 \AA)$ column with an inline VHP filter $(0.5 \mu \mathrm{m}$, stainless steel) from Upchurch Scientific. The mobile phase for the positive ESI-QTOF-MS consisted of A) $0.1 \%$ formic acid aqueous solution ( $\mathrm{v} / \mathrm{v}$ ) and $B$ ) acetonitrile. The mobile phase for the negative ESI-QTOF-MS consisted of A) $0.05 \%$ ammonium hydroxide aqueous solution ( $\mathrm{v} / \mathrm{v}$ ) and $B$ ) acetonitrile. The gradient elution profile was as follows: 0-4 $\min , 5 \% \mathrm{~B} ; 4-7 \mathrm{~min}, 5-10 \% \mathrm{~B} ; 7-20 \mathrm{~min}, 10-15 \% \mathrm{~B} ; 20-30 \mathrm{~min}$, $15-22 \%$ B; 30-35 min, 22-35\% B; 35-40 min, 35-50\% B; 40-45 min, 50-70\% B; 45-50 min, 70-90\% B; 50-52 min, 5\% B; 52-60 min, $5 \% \mathrm{~B}$. The flow rate was $0.4 \mathrm{~mL} / \mathrm{min}$. The column temperature was at $60^{\circ} \mathrm{C}$. The sample injection volume was $5.00 \mu \mathrm{L}$. Prior to sample analyses, the column was equilibrated with the mobile phase for at least $30 \mathrm{~min}$ at a flow rate of $0.4 \mathrm{~mL} / \mathrm{min}$.

The QTOF-MS was operated in both positive and negative ESI modes. The chromatographic and spectra data (.d) were acquired for each ESI mode using Agilent MassHunter Data Acquisition software (Version B.05.01). The operation parameters of the mass spectrometer were as follows: drying gas $\left(\mathrm{N}_{2}\right)$ temperature, $350^{\circ} \mathrm{C}$; drying gas flow rate, $10.0 \mathrm{~L} / \mathrm{min}$; nebulizer gas $\left(\mathrm{N}_{2}\right)$ pressure, $35 \mathrm{psi}$; sheath gas flow rate, $11.0 \mathrm{~L} / \mathrm{min}$; sheath gas $\left(\mathrm{N}_{2}\right)$ temperature, $325^{\circ} \mathrm{C}$; capillary voltage, $4000 \mathrm{~V}$; nozzle voltage, $500 \mathrm{~V}$; fragmentor voltage, $100 \mathrm{~V}$; skimmer voltage, $65 \mathrm{~V}$; octopole radio-frequency voltage, $750 \mathrm{~V}$; collision energy, 10, 20, and $40 \mathrm{eV}$. The data were acquired by auto MS/MS mode with an extended dynamic range ( $2 \mathrm{GHz}$ ). The MS scan range was $50-1000 \mathrm{~m} / \mathrm{z}$ at a scan rate 5 spectra/s. The MS/MS scan range was $50-1000 \mathrm{~m} / \mathrm{z}$ at a scan rate of 3 spectra/s with an isolation width at narrow $(\sim 1.3 \mathrm{~m} / \mathrm{z})$. To maintain the mass accuracy, the mass spectrometer was calibrated and tuned before analysis, and internal reference masses from the reference mass solution were used for real-time mass correction at $\mathrm{m} / \mathrm{z} 121.0508$ and 922.0098 for the positive ion mode, and $\mathrm{m} / \mathrm{z} 112.9885$ and 1033.9881 for the negative ion mode throughout the acquisition process. The reference mass solution was prepared using an Agilent API-TOF reference mass solution kit (Part No: G1969-85001).

\section{Method validation}

The specificity and reproducibility of the UPLC-QTOF-MS method were assessed by replicate measurements of samples of four Yinqiaosan preparation forms. PCA score plots were constructed using the acquired MS data, and the CVs were calculated on the concentrations of replicate measurements of 49 commonly identified compounds through global semiquantitative analysis.

\section{Data processing, constituent identification, and statistical analysis}

The data files (.d) of replicate measurements for four Yinqiaosan preparation forms acquired by Agilent MassHunter Data Acquisition software at the same ion polarity (i.e., either positive or negative ESI) were first assessed using Agilent MassHunter Qualitative Analysis software (Version: B.06.00) for the signal/noise level, and retention time and mass shifts with respect to the spiked IS, which were then processed using Agilent Profinder software (Version B.06.00) for batch recursive analysis. The data files were grouped into four preparation forms and subjected to molecular feature extraction by selecting a peak height threshold of 1000 counts, pos- sible ion adducts, isotope model of common organic molecules, charge states up to two, a retention time window of $0.10 \%+0.60$ min, a mass window of 20.00 ppm $+2.00 \mathrm{mDa}$ (for alignment of the IS in all runs with the same polarity), and a post-processing filter at 3 out of 3 replicate measurements for each Yinqiaosan preparation form.

Each molecular feature extracted data file (.cef) by Profinder software along with the corresponding data file (.d) by MassHunter Data Acquisition software was imported into Agilent MassHunter Qualitative Analysis software. Using “Find by Formula”, MS/MS data along with its MS and retention time data were extracted. The new data files (.cef) in the same ion polarity of replicate measurements of each Yinqiaosan preparation form were then imported into Agilent Mass Profiler Professional software (Version: B.13.1.1) for an METLIN AM database search and molecular formula generation. The selection of elements and limits for molecular formula generation were as follows: $\mathrm{C}(3-156), \mathrm{H}(0-180), \mathrm{O}(0-40), \mathrm{N}(0-$ 20), S (0-14), Cl (0-12), F (0-12), Br (0-10), P (0-9), and Si (0-15) [55]. The top five constituents with the highest scores were annotated, and cross-checked with the Traditional Chinese Medicine Integrated Database [56] and Traditional Chinese Medicine Systems Pharmacology Database and Analysis Platform [57] for final name assignments. The combined data file (.cef) of replicate measurements of each Yinqiaosan preparation form with the names of chemical constituents was saved for global semiquantitative analysis.

For multivariate data analysis, the molecular feature extracted data files (.csv) by the Profinder software were imported into MetaboAnalyst 4.0 [58] in terms of mass, retention time, and peak area. The mass tolerance and the retention time tolerance were set at 0.025 and $30 \mathrm{~s}$, respectively. Sample normalization was performed by the IS reference features (i.e., mass, retention time, and peak area), and the data were log transformed and autoscaled. PCA and PLS-DA were performed using the IS for normalization. PCA and PLS-DA score plots were constructed for similarity comparison of the chemical constituents and reproducibility assessment of the UPLC-QTOF-MS method as well as pattern recognition among the four Yinqiaosan preparation forms.

\section{Global semiquantitative analysis}

The data files (.d) with the same ion polarity of replicate measurements of each Yinqiaosan preparation form by the MassHunter Data Acquisition software and their corresponding combined data file (.cef) with the names of chemical constituents by the Mass Profiler Professional software were imported into Agilent MassHunter Quantitative Analysis software (Version: B.06.00). In the method setup task, the retention time window was set at $0.6 \mathrm{~min}$, etoposide-d3 ammonium adduct was chosen as the IS and flagged, other identified chemical constituents were chosen as targets relative to the IS, and proper ion polarity was chosen. After validating the method setup, global semiquantitative analysis was performed based on the peak area ratio of each individual target to the IS, and the quantitation data were exported as excel file for reporting. 


\section{Supporting Information}

The MS/MS spectra of the 49 chemical constituents commonly identified in all four Yinqiaosan preparation forms ( $\vee$ Fig. 1S), the common chemical constituents detected but unidentified in all four Yinqiaosan preparation forms ( $\triangleright$ Table 1S), and the unique constituents found in decoction, granule, pill, and tablet forms of Yinqiaosan ( $>$ Tables 2S-9S) are available as Supporting Information.

\section{Acknowledgements}

Yachun Shu would like to acknowledge the funding support of the China Natural Science Foundation (81503216) and Jiangsu Province (LGY2016017).

\section{Conflict of Interest}

The authors declare that they have no conflict of interest.

\section{References}

[1] Cheng CW, Wu TX, Shang HC, Li YP, Altman DG, Moher D, Bian ZX. CONSORT Extension for Chinese Herbal Medicine Formulas 2017: Recommendations, Explanation, and Elaboration (Traditional Chinese Version). Ann Intern Med 2017; 167: 112-121

[2] Fung FY, Linn YC. Developing traditional Chinese medicine in the era of evidence-based medicine: current evidences and challenges. Evid Based Complement Alternat Med 2015; 2015: 425037

[3] The Chinese Pharmacopoeia Commission. Pharmacopoeia of the People's Republic of China, Volume I. Beijing: China Medical Science Press. 2015

[4] The United States Pharmacopeial Convention. Pharmacopeial Convention Herbal Medicines Compendium. Available at: https://hmc. usp.org/monographs/all. Accessed February 20, 2020

[5] The European Pharmacopoeia Commission. European Pharmacopoeia, Volume I. Strasbourg, France: EDQM. 2017

[6] Ma XD, Fan YX, jin CC, Wang F, Xin CZ, Li P, Li HJ. Specific targeted quantification combined with non-targeted metabolite profiling for quality evaluation of Gastrodia elata tubers from different geographical origins and cultivars. J Chromatogr A 2016; 1450: 53-63

[7] Li W, Tang Y, Guo J, Shang E, Qian Y, Wang L, Zhang L, Liu P, Su S, Qian $D$, Duan J. Comparative metabolomics analysis on hematopoietic functions of herb pair Gui-Xiong by ultra-high-performance liquid chromatography coupled to quadrupole time-of-flight mass spectrometry and pattern recognition approach. J Chromatogr A 2014; 1346: 49-56

[8] Kim N, Ryu SM, Lee D, Lee JW, Seo EK, Lee JH, Lee D. A metabolomic approach to determine the geographical origins of Anemarrhena asphodeloides by using UPLC-QTOF MS. J Pharm Biomed Anal 2014; 92: 47-52

[9] Xie M. Formulaology. Beijing: People's Medical Publishing House 2007; 53-54

[10] Wu T. Analysis of Warm Diseases, Volume I. Beijing: People's Medical Publishing House 2017; 17-19

[11] Chen Y], Zhu XX, Cai BC, Wang Q, Shu YC. Comparative study on effects of Yinqiao powder decocted later at different time against influenza a virus in body. CJTCMP 2015; 30: 4048-4052
[12] Yu Y, Zhang G, Huang HL, Lu Z, Qiu DW, Han T, Tang JQ. Meta-analysis of the clinical effects of Yinqiao decoction on hand-foot-mouth disease. JETCM 2016; 25: 590-593

[13] Chen QY. The Clinical and Basic Study of Yinqiao Powder for Acute Viral Pharyngitis [doctoral dissertation]. Guangzhou: Guangzhou University of Chinese Medicine. 2013

[14] Xi YL, Hui HY, Li DT, Deng Z]. Clinical efficacy of Yinqiao powder against pediatric pneumonia. Chin Hosp Pharm J 2016; 36: 571-574

[15] Li YQ. Experience of Yinqiao Powder for the Treatment of Children with Acute Tonsillitis. Chinese Community Doctors 2016; 32: 118120

[16] Duan SH, Liu MS. Study on adult measles of treatment effect with Yinqiao powder. Chinese Journal of Basic Medicine in Traditional Chinese Medicine 2014; 20: 1150-1151

[17] Shu YC, Qin KM, Chen Y], Zhu XX, Cai H, Lu TL, Li H, Cai BC. Study on the different decocting methods on Yinqiaosan decoction of anti-inflammatory and antipyretic effects. CJTCMP 2013; 28: 1413-1418

[18] Wang C, Cao B, Liu QQ, Zou ZQ, Liang ZA, Gu L, Dong JP, Liang LR, Li XW, Hu K, He XS, Sun YH, An Y, Yang T, Cao ZX, Guo YM, Wen XM, Wang YG, Liu YL, Jiang LD. Oseltamivir compared with the Chinese traditional therapy Maxingshigan-Yinqiaosan in the treatment of H1N1 influenza: A randomized trial. Ann Intern Med 2011; 155: 217-225

[19] Wang JJ, Zhang L, Guo Q, Kou JP, Yu BY, Gu DH. Quantitative analysis of seven phenolic acids in eight Yinqiaojiedu serial preparations by quantitative analysis of multi-components with single-marker. Acta Pharmaceutica Sinica 2015; 50: 480-485

[20] Hao M, Lu TL, Mao CQ, Wang QH, Su LL, Zhang J. Study on quality control of Chinese herbal pieces based on Q-marker of Chinese materia medica. Chinese Traditional and Herbal Drugs 2017; 48: 1699-1708

[21] Want E], Wilson ID, Gika H, Theodoridis G, Plumb RS, Shockcor ], Holmes E, Nicholson JK. Global metabolic profiling procedures for urine using UPLC-MS. Nat Protoc 2010; 5: 1005-1018

[22] Wang X, Hao O, Wang W, Ying X, Wang H. Evaluation of the use of different solvents to extract the four main components of Yinqiaosan and their in vitro inhibitory effects on influenza-A virus. Kaohsiung J Med Sci 2010; 26: 182-191

[23] Shu YC, Qin KM, Cai H, Liu X, Yin FZ, Zhang JZ, Cai BC. Study on the optimal traditional decoction process for Yinqiao San by orthogonal experiments. CJTCMP 2012; 27: 2651-2655

[24] Chiruvella KK, Mohammed A, Dampuri G, Ghanta RG, Raghavan SC. Phytochemical and antimicrobial studies of methyl angolensate and luteolin-7-O-glucoside isolated from callus cultures of Soymida febrifuga. Int J Biomed Sci 2007; 3: 269-278

[25] Hu C, Kitts DD. Luteolin and luteolin-7-O-glucoside from dandelion flower suppress iNOS and COX-2 in RAW264.7 cells. Mol Cell Biochem 2004; 265: 107-113

[26] Zang Y, Igarashi K, Li Y. Anti-diabetic effects of luteolin and luteolin7-O-glucoside on KK-A(y) mice. Biosci Biotechnol Biochem 2016; 80: $1580-1586$

[27] Wu YT, Cai MT, Chang CW, Yen CC, Hsu MC. Bioanalytical method development using liquid chromatography with amperometric detection for the pharmacokinetic evaluation of forsythiaside in rats. Molecules 2016; 21: E1384

[28] Chen L, Lin L, Dong Z, Zhang L, Du H. Comparison of neuroprotective effect of Forsythia suspensa leaf extract and forsythiaside, one of its metabolites. Nat Prod Res 2017; 8: 1-4

[29] Pan X, Cao X, Li N, Xu Y, Wu Q, Bai J, Yin Z, Luo L, Lan L. Forsythin inhibits lipopolysaccharide-induced inflammation by suppressing JAK-STAT and p38 MAPK signalings and ROS production. Inflamm Res 2014; 63: 597-608 
[30] Huang X, Wang Y, Ren K. Protective effects of liquiritin on the brain of rats with Alzheimer's disease. West Indian Med J 2015; 64: 468-472

[31] Zhang Y, Zhang L, Zhang Y, Xu J], Sun LL, Li SZ. The protective role of liquiritin in high fructose-induced myocardial fibrosis via inhibiting NF-KB and MAPK signaling pathway. Biomed Pharmacother 2016; 84: 1337-1349

[32] Hayashi K, Narutaki K, Nagaoka Y, Hayashi T, Uesato S. Therapeutic effect of arctiin and arctigenin in immunocompetent and immunocompromised mice infected with influenza A virus. Biol Pharm Bull 2010; 33: 1199-1205

[33] Lee S, Shin S, Kim H, Han S, Kim K, Kwon J, Kwak JH, Lee CK, Ha NJ, Yim D, Kim K. Anti-inflammatory function of arctiin by inhibiting COX-2 expression via NF-KB pathways. J Inflamm (Lond) 2011; 8: 1-9

[34] Matsuzaki Y, Koyama M, Hitomi T, Yokota T, Kawanaka M, Nishikawa A, Germain D, Sakai T. Arctiin induces cell growth inhibition through the down-regulation of cyclin D1 expression. Oncol Rep 2008; 19: 721-727

[35] Hirose M, Yamaguchi T, Lin C, Kimoto N, Futakuchi M, Kono T, Nishibe $S$, Shirai T. Effects of arctiin on PhIP-induced mammary, colon and pancreatic carcinogenesis in female Sprague-Dawley rats and MelQx-induced hepatocarcinogenesis in male F344 rats. Cancer Lett 2000; 155: 79-88

[36] Chen J, Kudo H, Kan K, Kawamura S, Koseki S. Growth inhibitory effect of d-tryptophan on Vibrio spp. in shucked and live oysters. Appl Environ Microbiol 2018; 84: e01543-18

[37] Cheng Z, Yuan X, Qu Y, Li X, Wu G, Li C, Zu X, Yang N, Ke X, Zhou J, Xie $\mathrm{N}$, Xu X, Liu S, Shen Y, Li H, Zhang W. Bruceine D inhibits hepatocellular carcinoma growth by targeting $\beta$-catenin/jagged1 pathways. Cancer Lett 2017; 403: 195-205

[38] Hughes AD, Hering S, Bolton TB. Evidence that agonist and antagonist enantiomers of the dihydropyridine PN 202-791 act at different sites on the voltage-dependent calcium channel of vascular muscle. $\mathrm{Br}$ ] Pharmacol 1990; 101: 3-5

[39] Shanmugam MK, Shen H, Tang FR, Arfuso F, Rajesh M, Wang L, Kumar AP, Bian J, Goh BC, Bishayee A, Sethi G. Potential role of genipin in cancer therapy. Pharmacol Res 2018; 133: 195-200

[40] Kim BR, Jeong YA, Na Y], Park SH, Jo M], Kim JL, Jeong S, Lee SY, Kim HJ, Oh SC, Lee DH. Genipin suppresses colorectal cancer cells by inhibiting the Sonic Hedgehog pathway. Oncotarget 2017; 8: 101952-101964

[41] Lee SY, Kim H], Oh SC, Lee DH. Genipin inhibits the invasion and migration of colon cancer cells by the suppression of HIF- $1 \alpha$ accumulation and VEGF expression. Food Chem Toxicol 2018; 116: 70-76

[42] Choi EM, Suh KS, Lee YS. Liquiritigenin restores osteoblast damage through regulating oxidative stress and mitochondrial dysfunction. Phytother Res 2014; 28: 880-886

[43] Xie XW. Liquiritigenin attenuates cardiac injury induced by high fructose-feeding through fibrosis and inflammation suppression. Biomed Pharmacother 2017; 86: 694-704
[44] Bae GD, Park EY, Baek DJ, Jun HS, Oh YS. Liquiritigenin prevents palmitate-induced beta-cell apoptosis via estrogen receptor-mediated AKT activation. Biomed Pharmacother 2018; 101: 348-354

[45] Miell J, Dhanjal P, Jamookeeah C. Evidence for the use of demeclocycline in the treatment of hyponatraemia secondary to SIADH: a systematic review. Int J Clin Pract 2015; 69: 1396-1417

[46] Galski H, Lazarovici P, Gottesman MM, Murakata C, Matsuda Y, Hochman J. KT-5720 reverses multidrug resistance in variant $\$ 49$ mouse lymphoma cells transduced with the human MDR1 CDNA and in human multidrug-resistant carcinoma cells. Eur J Cancer 1995; 31A: 380-388

[47] Galski H, Sivan H, Lazarovici P, Nagler A. In vitro and in vivo reversal of MDR1-mediated multidrug resistance by KT-5720: implications on hematological malignancies. Leuk Res 2006; 30: 1151-1158

[48] Jeong MY, Park J, Youn DH, Jung Y, Kang J, Lim S, Kang MW, Kim HL, So HS, Park R, Hong SH, Um JY. Albiflorin ameliorates obesity by inducing thermogenic genes via AMPK and PI3K/AKT in vivo and in vitro. Metabolism 2017; 73: 85-99

[49] Zhang L, Cheng L, Hong J. The clinical use of cetirizine in the treatment of allergic rhinitis. Pharmacology 2013; 92: 14-25

[50] Nayak AS, Berger WE, LaForce CF, Urdaneta ER, Patel MK, Franklin KB, Wu MM. Randomized, placebo-controlled study of cetirizine and loratadine in children with seasonal allergic rhinitis. Allergy Asthma Proc 2017; 38: 222-230

[51] Hu Y, Yu K, Wang G, Zhang D, Shi C, Ding Y, Hong D, Zhang D, He H, Sun L, Zheng JN, Sun S, Qian F. Lanatoside C inhibits cell proliferation and induces apoptosis through attenuating Wnt/ $\beta$-catenin/c-Myc signaling pathway in human gastric cancer cell. Biochem Pharmacol 2018; 150: 280-292

[52] Chao MW, Chen TH, Huang HL, Chang YW, HuangFu WC, Lee YC, Teng CM, Pan SL. Lanatoside C, a cardiac glycoside, acts through protein kinase $C \delta$ to cause apoptosis of human hepatocellular carcinoma cells. Sci Rep 2017; 7: 46134

[53] Hopkins AL. Network pharmacology: the next paradigm in drug discovery. Nat Chem Biol 2008; 4: 682-690

[54] Chen YL, Zhang YL, Dai YC, Tang ZP. Systems pharmacology approach reveals the anti-inflammatory effects of ampelopsis grossedentata on dextran sodium sulfate-induced colitis. World J Gastroenterol 2018; 24: 1398-1409

[55] Kind T, Fiehn O. Seven golden rules for heuristic filtering of molecular formulas obtained by accurate mass spectrometry. BMC Bioinformatics 2001; 8: 105

[56] Center for Bioinformatics and Computational Biology at East China Normal University, Traditional Chinese Medicine Integrated Database. Available at: http://119.3.41.228:8000/tcmid. Accessed February 20, 2020

[57] Wang Y. Traditional Chinese Medicine Systems Pharmacology Database and Analysis Platform. Available at: http://tcmspw.com. Accessed February 20, 2020

[58] Xia J. MetaboAnalyst 4.0. Available at http://www.metaboanalyst.ca. Accessed February 20, 2020 